Anette Müller

Baustoffrecycling.

Entstehung - Aufbereitung

- Verwertung

Die Notwendigkeit, Stoffkreisläufe im Bauwesen zu etablieren, ist unbestritten. Das Wissen dazu ist an vielen Stellen vorhanden und wird im vorliegenden Buch zusammengefasst und systematisiert. Nach einem allgemeinen Überblick zu den Anfallmengen, Verwertungsquoten und Einsatzgebieten von Recycling-Baustoffen wird darauf eingegangen, auf welchen Aufbereitungsschritten das Recycling gegenwärtig basiert und welche Möglichkeiten der Einflussnahme auf die Produkteigenschaften bestehen. Des Weiteren werden RecyclingBaustoffe charakterisiert und deren Einsatzgebiete dargestellt. Ausgangspunkt ist immer der ursprüngliche Baustoff, der sich später im Bauabfall wiederfindet. Dabei liegt der Schwerpunkt auf den bautechnischen Eigenschaften. Die umwelttechnischen Aspekte, die seit Jahren die Diskussion bestimmen, sind im notwendigen Umfang aufgezeigt. Den Abschluss des Buches bildet ein Kapitel, das neue Entwicklungen in der Aufbereitungstechnik präsentiert und die Potenziale von Bauabfällen als Rohstoffquelle analysiert.

\section{Roman Teschner}

\section{Glasfasern}

Das Buch stellt eine wichtige Datenquelle von physikalisch-chemischen Eigenschaften der Fasergläser, Glasfasern und der für ihre Herstellung eingesetzten Glasrohstoffe und technologischen Prozessparameter dar. Es hilft dem Leser, die Eigenschaften der Fasergläser und Glasfasern besser zu verstehen, um sie im Herstellungsprozess und in der Weiterverarbeitung optimal einzusetzen. Die Herstellprozesse der verschiedenen Glasfaserprodukte wurden in einer kompakten Form kurz und verständlich dargestellt. Das Buch kann sowohl den Glasfachleuten, den Compositsspezialisten, die in der Forschung und Entwicklung bzw. in der Produktion tätig sind, als auch Studierenden der Fachbereiche Glaschemie, Glastechnologie und Werkstoffwissenschaften sehr behilflich sein. Das Buch wurde von einem Praktiker geschrieben, der die Faserglasentwicklung und die Glasfaserproduktion sowie Faserziehprozesse über 20 Jahre aktiv begleitet und erforscht hat.

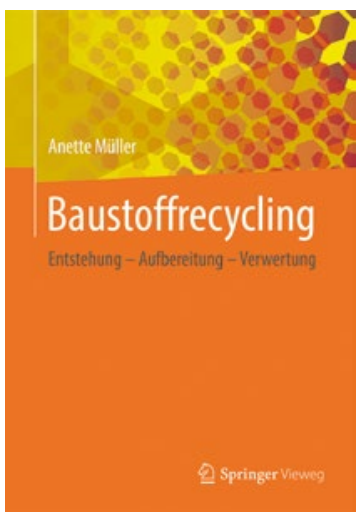

Springer Fachmedien

Wiesbaden, 2018, 336 Seiten,

Softcover,

ISBN: 978-3-658-22987-0, 29,99 Euro auch als E-Book erhältlich für 22,99 Euro unter www.springer.com/booksellers

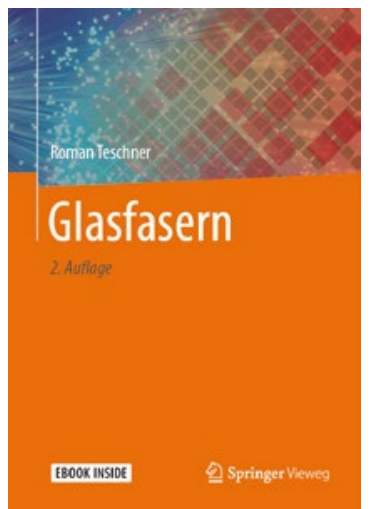

Springer Berlin Heidelberg, 2019, 2. Auflage, 233 Seiten,

Hardcover,

ISBN: 978-3-662-58370-8, 70,00 Euro auch als E-Book erhältlich für 54,99 Euro unter www.springer.com/booksellers 